\title{
SISTEMAS DE INFORMAÇÃO EM SAÚDE: A IMPORTÂNCIA DO SOFTWARE LIVRE E DA MODELAGEM MULTINÍVEL
}

\author{
Health Information Systems: The Importance of Open Source and Multilevel Modeling
}

\author{
Luciana Tricai Cavalini' ${ }^{1}$ Timothy Wayne Cook ${ }^{2}$
}

Resumo A informação em saúde apresenta complexidades significativas em termos espaço-temporais e de domínio, o que traz desafios para o desenvolvimento de sistemas de informação em saúde centrados no paciente, interoperáveis e semanticamente coerentes. Este artigo de revisão sustenta a ideia de que a abordagem de modelagem multinível é essencial para garantir a interoperabilidade a nível semântico, mas uma verdadeira interoperabilidade só é obtida com a adoção de padrões abertos, e implementações de código aberto são necessárias para promover a concorrência com base na qualidade do software. As especificações Multilevel Healthcare Information Modeling (MLHIM) são apresentadas, como uma referência para a modelagem multinível totalmente baseada em software livre, e são sugeridas boas práticas para o desenvolvimento de aplicativos para a área de saúde baseados em modelagem multinível e software livre.

Palavras-chave: Telemedicina, Telessaúde, Atenção a Saúde

Abstract Health information features significant spatial-temporal and domain complexities, which brings challenges to the implementation of patient-centered, interoperable and semantically coherent healthcare information systems. This position paper supports the idea that the multilevel modeling approach is essential to ensure interoperability at the semantic level, but true interoperability is only achieved by the adoption of open standards, and open source implementations are needed for promote competition based on software quality. The Multilevel Healthcare Information Modeling (MLHIM) specifications are presented as the fully open source multilevel modeling reference implementation, and best practices for the development of multilevel-based open source healthcare applications are suggested.

Keywords: Medical Informatics, Software Design, Information Systems

\section{Introdução}

As informações relacionadas à saúde humana são inerentemente complexas nas interseções de espaço, tempo e domínio'. Esta complexidade impede a viabilidade de um registro eletrônico de saúde monolítico, uma vez que vários tipos de informação, provenientes de um grande número de diferentes aplicações, podem ser necessários, a qualquer momento para o atendimento de um paciente ${ }^{2}$. Assim, a interoperabilidade semântica é crucial para o registro de informações em saúde em aplicativos específicos, que precisam ser sincronizados

\footnotetext{
1. Professora Adjunto do Departamento de Tecnologias de Informação e Educação em Saúde da Faculdade de Ciências Médicas da UERJ - Doutorado em Saúde Coletiva pelo Instituto de Medicina Social da UERJ; 2. Colaborador Internacional do Laboratório “Multilevel Healthcare Information Modeling" (LA-MLHIM), Associado ao Instituto Nacional de Ciência e Tecnologia - Medicina Assistida por Computaçao Científica - Mestre em Health Informatics pela University of Central Lancashire. Endereço Eletrônico: lutricav@lampada.uerj.br (Luciana Tricai Cavalini).
} 
a grandes bases de dados hospitalares. Esta é a maneira ideal de fornecer informações úteis a partir do local de cuidado, para os todos os demais serviços de saúde onde o paciente será acolhido, de forma oportuna ${ }^{3}$.

Em relação à saúde pública, o tempo de resposta típico atual para se conhecer a situação de saúde de uma população pode ser de semanas ou até meses, enquanto especialistas se debruçam sobre os dados e tentam mesclar fragmentos de informação provenientes de diversos sistemas de informação, e mesmo de formulários de papel, em algo significativo 4 .

Ao se basear os sistemas de informação em um modelo de informação comum e usando uma abordagem baseada em restrições ao modelo comum para definir os componentes do conhecimento, é possível alcançar a interoperabilidade semântica e prover informações, quase em tempo real, sobre o estado de saúde de uma população e de seus indivíduos, para que as medidas necessárias possam ser tomadas, de forma a atender às necessidades em saúde. Essa abordagem também ajuda a capacitar os desenvolvedores de aplicativos em nível local, para que estes possam desenvolver sistemas de informação em saúde adequados às necessidades específicas locais, sem perda da coerência semântica e da interoperabilidade com outros serviços locais e com os níveis mais elevados do sistema de saúde ${ }^{5}$.

As desigualdades em saúde não serão facilmente resolvidas pelo exercício de uma medicina do século 21 com base em sistemas de informação do século 206. Não há obstáculos remanescentes relacionados a hardware, incluindo computação móvel e medicina pervasiva, mas sistemas de informação com base em modelos de dados tradicionais não estão equipados para lidar com as significativas complexidades espaciais e temporais da informação em saúde ${ }^{7}$. Isto ocorre porque tais sistemas não são interoperáveis e têm altos custos de manutenção. Estes problemas reduzem a utilidade dos atuais sistemas de informação para o enfren- tamento das situações emergentes e dinâmicas vividas nos serviços de saúde contemporâneos ${ }^{8,9}$. De fato, o desenvolvimento de aplicações de saúde é um desafio complexo, especialmente dado o grande número de conceitos em constante evolução, o que torna difícil se chegar a um consenso sobre qualquer conceito ${ }^{10}$.

Algumas soluções para estes problemas têm sido propostas ao longo das últimas duas décadas. No entanto, a solução mais adequada para as características específicas das informações de saúde envolve a separação entre o modelo de domínio e a persistência dos dados. Esta abordagem, denominada modelagem multinível, propõe o desenvolvimento de sistemas de informação baseados na definição de pelo menos dois níveis: o Modelo de Referência, que define os tipos genéricos de dados e de estruturas de dados, e um Modelo de Domínio, definido por restrições as Modelo de Referência"1.

Sistemas de informação em saúde baseados em modelagem multinível são facilmente interoperáveis e podem ser implementados em qualquer hardware. A adoção de um Modelo de Referência comum e de um Modelo de Domínio compartilhado entre sistemas diferentes permite o estabelecimento uma interface transparente entre os mesmos, assim como intercâmbio de dados para sistemas de informação geográfica e para ferramentas de análise estatística, facilitando a análise das informações coletadas a partir de vários sistemas remotos ${ }^{12}$.

Hoje em dia, há especificações de modelagem multinível para sistemas de informação de saúde abertamente disponíveis e comprovadas em software. Com base nessas especificações, é possível desenvolver sistemas de informação em saúde centrados no cidadão, com a capacidade de registro de dados longitudinais ${ }^{13}$.

Além disso, ferramentas de apoio à decisão e de geração de relatórios padronizados podem ser incorporadas aos sistemas, sem prejuízo da interoperabilidade semântica em qualquer nível, dado que o desenvolvi- 
mento de algoritmos de apoio à decisão baseados em um modelo de domínio comum permitem a reutilização de regras de decisão em diferentes implementações. Assim, no momento do cuidado, medidas de controle podem ser implementadas imediatamente, permitindo uma maior eficácia dos cuidados de saúde e, ao nível da administração, grandes áreas geográficas podem ser monitorizadas, permitindo a identificação de áreas de prioridade para intervenção ${ }^{14}$.

No entanto, apesar de suas vantagens técnicas, soluções baseadas em modelagem multinível ainda não foram amplamente implementadas em serviços de saúde reais, com exceção de alguns poucos projetos acadêmicos registrados na literatura científica ${ }^{15-17}$.

Há um aspecto que é essencial, mas que é raramente abordado sobre a interoperabilidade dos sistemas de informação em saúde, que está relacionado à modalidade de licenciamento do software. Na verdade, o modelo de negócios clássico das empresas de software proprietário pode ser considerado hostil à interoperabilidade, dado que a competição entre as empresas tem o objetivo de estabelecer a hegemonia ou o monopólio, a fim de concentrar o capital, o que é baseado no sigilo do código fonte do software. Na verdade, pode-se afirmar, não há interoperabilidade comprovada sem o desenvolvimento de, ao menos, especificações abertas, uma vez que é necessário que um sistema seja compatível com as características do outro sistema que estão relacionadas com a interoperabilidade, o que só pode ser alcançado se os sistemas forem desenvolvidos com base em um conjunto comum de especificações. Assim, expandindo o argumento, é possível deduzir que a condição completa da interoperabilidade entre todos os sistemas somente é possível se eles compartilharem um conjunto comum de especificações, ao menos ao nível da troca extratos de informação. Portanto, a plena interoperabilidade exige padrões abertos e software de código aberto ${ }^{18,19}$.
Este modelo de negócios não revelou quaisquer diferenças quando aplicado à saúde. Na verdade, afirma-se que os padrões abertos facilitam a concorrência entre software livre e software proprietário, uma vez que permite a competição entre diferentes implementações da mesma especificação ${ }^{20}$. Esta é uma questão fundamental relacionada à qualidade de software, o que é fundamental na área da saúde, já que a qualidade do software está diretamente relacionada à qualidade do atendimento ${ }^{21}$.

Levando-se em conta a centralidade dos princípios do software livre e da modelagem multinível para garantir o desenvolvimento sistemas de informação em saúde semanticamente coerentes de alta qualidade, centrados no cidadão e interoperáveis, o objetivo deste artigo é descrever as características essenciais de uma especificação aberta para modelagem multinível de sistemas de informação em saúde e propor um conjunto de boas práticas para o desenvolvimento de aplicativos em saúde com base nessas especificações.

\section{Modelagem Multinível}

\subsection{Resumo das Especificações}

As especificações Multilevel Healthcare Information Modeling (MLHIM) são um conjunto de especificações totalmente abertas para o desenvolvimento de sistemas de informação em saúde com base em modelagem multinível. A documentação das especificações MLHIM é publicada no formato Open Document Format (ODF) e está disponível em http://www.mlhim.org.

As escolhas tecnológicas para o desenvolvimento das especificações MLHIM foram feitas em função da natureza distribuída e diversificada dos sistemas de informação de saúde; assim, o seu objetivo é a interoperabilidade, e a padronização é o caminho. A base das especificações MLHIM é o modelo dual proposto pela openEHR Foundation ${ }^{22} \mathrm{e}$ alguns aspectos da Norma ISO 21090 relativos aos tipos 
de dados em saúde. Estes padrões foram articulados em uma única especificação, com o propósito específico de criar um caminho para a interoperabilidade semântica entre diferentes aplicações de saúde, incluindo sistemas legados.

Nas especificações MLHIM, as classes do Modelo de Referência são persistentes e devem ser mantidas tão estáveis quanto possível ao longo do tempo. No Modelo de Domínio, as definições de restrição às classes do Modelo de Referência fornecem a interpretação semântica dos objetos, armazenados de acordo com o Modelo de Referência.

A ideia por trás da modelagem multinível é que as mudanças na estrutura e nas regras de inferência são refletidas sobre o Modelo de Domínio e não sobre o Modelo de Referência. Assim, as solicitações de alteração nos mecanismos de persistência dos sistemas de informação são reduzidas. Além disso, os Modelos de Domínio são criados e editados por especialistas da área, o que evita a necessidade de interpretação do conhecimento extraído de uma interação ad hoc entre o especialista e o desenvolvedor do software. Uma vez que o especialista de domínio é responsável por modelar o conhecimento, os conceitos são definidos completamente e com precisão.

\subsection{Modelagem do Conhecimento}

As especificações MLHIM adotam a linguagem XML Schema Definition (XSD) para a elaboração dos Modelos de Domínio, que são denominados Concept Constraint Definitions (CCDs). Um CCD é um arquivo XSD que expressa um conceito de saúde definido. Este conceito é expresso no CCD como definições de restrição às classes do Modelo de Referência MLHIM.

XML é considerada como a solução mais amplamente adotada para a interoperabilidade e a coerência semântica entre sistemas e, portanto, a fim de se ajustar aos seus propósitos, XML deve ser um padrão aberto ${ }^{23}$. Existem algumas publicações a partir de 2005 sobre o uso de XSDs na área de informática em saúde como uma tentativa de realizar padronizações a posteriori de tipos de dados e metadados ${ }^{24,25}$, desenvolvimento de modelos para documentos estruturados ${ }^{26,27}$, ou qualquer combinação das técnicas acima mencionadas ${ }^{28-30}$, o que pode ser entendido como a busca de soluções para promover a troca de dados entre sistemas de informação baseados em modelagem tradicional de um nível.

Alguns desses estudos adotam o princípio de eleição do especialista de domínio como o autor da modelagem do conhecimento ${ }^{31}$. Embora essa abordagem resolva parte da perda semântica derivada de interações ad hoc entre o especialista de domínio e desenvolvedor do sistema, ela ainda não garante a coerência semântica e a interoperabilidade, nestes casos de tentativas de troca de dados entre aplicações baseadas em modelos de um nível.

Por outro lado, a utilização de XSDs para modelagem baseada no modelo multinível da Norma ISO 13606 padrão foi testado e validado por Rinner et al. ${ }^{32}$, permitindo a validação semântica de Modelos de Domínio, condicionais para a definição de uma "validação totalmente genérica" fornecida pelo Modelo de Referência. Por outro lado, os autores descrevem as dificuldades técnicas em relação às restrições às classes Modelo de Referência da ISO 13606 que são necessárias para expressar um dado conceito de saúde. Isto sugere a necessidade de desenvolvimento de bons editores de Modelos de Domínio (arquétipos openEHR ou ISO 13606, e CCDs MLHIM), o que é uma preocupação comum de projetos de modelagem multinível ${ }^{33,34}$.

Nas especificações MLHIM, os Modelos de Domínio são primeiramente elaborados em mapas mentais, que são comprovadamente eficientes como ferramentas de definição de conceitos $^{35}$. O software livre XMind (XMind Ltd.), um editor mapas mentais, foi adotado para elaborar um template (disponível em http://www.hkcr.net/tools) que representa graficamente as classes do Modelo de 
Referência MLHIM como tópicos, e os atributos das classes como subtópicos. O relacionamento de herança entre as classes é representado como relacionamentos entre tópicos. Assim, em vez de usar os tópicos do mapa mental para restringir diretamente os conceitos, eles são definidos pela combinação das classes que são necessárias para a definição da estrutura de dados um conceito específico, e a definição de restrições sobre os atributos dessas classes são expressos nos subtópicos. $\mathrm{O}$ arquivo XMind resultante informa a elaboração de um CCD no Constraint Definition Designer (CDD), um aplicativo em software livre baseado em Python, que deve produzir um arquivo XSD válido de acordo com o arquivo XSD do Modelo de Referência MLHIM (disponível em https://launchpad.net/mlhim-specs). Além disso, CCDs podem ser combinados com outros CCDs para informar o desenvolvimento de interfaces gráficas de usuário para sistemas de informação e aplicativos biomédicos baseados nas especificações MLHIM.

\section{Desenvolvimento de Sistemas}

Esta seção propõe um conjunto de boas práticas para o desenvolvimento de sistemas de informação em saúde baseados em modelagem multinível. O raciocínio subjacente aqui expresso é de que a interoperabilidade e a coerência semântica são asseguradas por especificações abertas baseadas em modelos multinível, tais como as especificações MLHIM, que incluem um Modelo de Referência genérico e estável, assim como as regras para as definições de restrição do Modelo de Referência necessárias para a modelagem de qualquer conceito em saúde.

Quaisquer outras características específicas, tais como a combinação de CCDs em modelos de dados maiores, a definição de interfaces gráficas de usuário, a escolha da linguagem de programação orientada a objeto, a escolha do banco de dados SQL, Não-SQL ou orientado a objeto para a persistência dos dados, e os algoritmos de consulta aos dados, são consideradas opções de implementação e não interferem nos aspectos técnicos relativos à interoperabilidade semântica, já tratadas de uma forma abrangente pelas especificações de modelagem multinível.

No entanto, a fim de garantir que a interoperabilidade e a coerência semânticas sejam atingidas pelos sistemas de informação baseados em modelagem multinível, é necessário o desenvolvimento de software de alta qualidade. Com base nesse raciocínio, um conjunto não exaustivo de boas práticas para o desenvolvimento de sistemas de informação em saúde baseados em modelagem multinível é apresentado abaixo.

\subsection{Plataforma de Desenvolvimento e Mecanismo de Persistência}

As especificações MLHIM são desenvolvidas em XSDs de forma a permitir a conversão de seu código para as principais linguagens orientadas a objeto, como Java, Python e Ruby. Este código-fonte pode ser incorporado a muitas plataformas de desenvolvimento de aplicativos baseados nas linguagens de programação mencionadas. Isto permitindo uma maior adoção das especificações, uma vez que uma plataforma de desenvolvimento de aplicativos específica não se torna uma curva de aprendizagem adicional para os programadores.

Normalmente, a plataforma de desenvolvimento de aplicativos escolhida orienta a escolha do tipo de banco de dados a ser adotado para a persistência de dados. É importante notar que, como as especificações MLHIM são orientados a objeto, a escolha mais óbvia seria um banco de dados orientado a objeto para a persistência de dados. No entanto, bancos de dados orientados a objeto podem apresentar problemas de desempenho ou de consulta. Levando esta dificuldade técnica em consideração, bancos de dados SQL ou Não-SQL pode ser adotados, a fim de contornar 
essas complexidades técnicas em aplicações da vida real, embora a persistência de dados em aplicações baseadas em MLHIM é trivial se um banco de dados orientado a objetos for escolhido.

\subsection{Camada de Comunicação}

A camada de comunicação deve ser construída para uso por qualquer componente de software baseado em tecnologia distribuída, disponível em linguagens de programação orientadas a objeto, operando em redes distribuídas ou sem fio. A associação entre as camadas de comunicação e de modelagem de dados pode ser feita através de uma abordagem orientada a modelo. Nesta abordagem, a arquitetura geral do sistema é especificada em um nível alto, usando uma Architecture Definition Language (ADL), implícita ou explicitamente anotada com os CCDs utilizados na camada de dados. Isto permite o desenvolvimento de interfaces gráficas de usuário, que podem assim ser persistidas, e posteriormente comunicadas através da rede, a partir de cada componente do sistema. Como sugestão, a biblioteca Acme ADL (disponível em http://www.cs.cmu.edu/ acme/) pode ser usada como base para a construção de geradores de código para diferentes partes da aplicação.

\subsection{Interface de Sistemas}

A adoção de modelagem multinível para o desenvolvimento de aplicações de saúde traz uma grande flexibilidade para os desenvolvedores de aplicativos. Não há mais nenhuma necessidade de desenvolvimento de um sistema de informação em saúde monolítico para o registro completo da saúde de um indivíduo, independente do tamanho e complexidade dos serviços de saúde acessados. A modelagem multinível permite o desenvolvimento de aplicativos específicos, incluindo aplicativos para o registro de dados de pesquisas científicas, mesmo que os CCDs não sejam os mesmos.
Assim, os dados podem ser trocados entre quaisquer aplicativos, e os extratos de dados de todos ainda são válidos para os demais. No entanto, algumas instituições podem exigir um maior nível de coesão do sistema, devido às especificidades de seu fluxo de trabalho, o que é comum em prontuários eletrônicos hospitalares.

Sendo assim, sugere-se a adoção dos princípios de uma Arquitetura Orientada para Serviços (Service Oriented Architecture - SOA), permite uma melhor articulação entre diferentes linguagens de programação e plataformas de desenvolvimento, o que é necessário, uma vez que aplicativos baseados em modelagem multinível podem ser desenvolvidos em qualquer linguagem orientada a objeto. Além disso, a SOA torna mais fácil a gestão de escalabilidade, reutilização, distribuição e armazenamento de aplicações. Assim sugere-se a adoção do estilo arquitetural Representational State Transfer (REST), mediante o uso de bibliotecas de código aberto (tais como Restfulie e RIP) para o desenvolvimento de clientes e servidores baseados em REST com a portabilidade para as principais linguagens orientadas a objeto. Essas bibliotecas permitem fracionamento do código, de modo que se cria um serviço muito flexível, capaz de evoluir com alterações mínimas no lado do cliente. Assim, é possível compatibilizar diferentes aplicações desenvolvidas em diferentes linguagens e plataformas de uma maneira custo-efetiva, reduzindo riscos e custos associados a tarefas tradicionais de integração de sistemas.

\subsection{Sistema de Apoio à Decisão}

O C Language Inference Processing System (CLIPS) é proposto como mecanismo de inferência para o desenvolvimento de algoritmos de apoio à decisão em sistemas de informação baseados em modelagem multinível. O CLIPS proporciona benefícios importantes para o desenvolvimento motores de apoio à decisão em sistemas de saúde, pelas razões apresentadas a seguir. 
Em primeiro lugar, CLIPS suporta um encadeamento prospectivo (ou guiado pelos dados) no modo de processamento de regras de inferência, o que significa que, sempre que surjam novos dados, todos estados disponíveis do sistema são verificados novamente. Adicionalmente CLIPS fornece uma robusta interface programação de aplicativos em $\mathrm{C} / \mathrm{C}++$, que permite, para o processamento de uma regra, a adição de rotinas que são interativas com o usuário, além da gestão de dependências entre as ações de controle dinâmico. Ambos os recursos são essenciais para as regras de modelagem de cenários dinâmicos, tais como os encontrados no setor saúde. Além disso, CLIPS suporta múltiplas camadas de persistência, permitindo um desenvolvimento de sistemas totalmente orientados a objeto, o que é essencial para sistemas de informação baseados em modelagem multinível.

\subsection{Processamento de Dados e Business Intelligence}

Para a elaboração de relatórios com base em dados agregados a partir do nível local, em direção aos níveis regionais e nacional, sugere-se que sejam baseadas em padrões tais como as especificações Statistical Data and Metadata Exchange - Health Domain (SDMX-HD), que têm sido desenvolvidas sob os auspícios da Organização Mundial de Saúde (OMS) para a normalização dos formatos de dados agregados, de modo a facilitar a elaboração de estatísticas e indicadores de saúde padronizados. Estas especificações estão inspirando alguns dos requisitos da norma ISO 14639. Para a execução de tarefas de Business Inteligence (BI), soluções em software livre, como a ferramenta de BI Pentaho (http://www.pentaho.com) são sugeridas para a pré-definição de relatórios gerenciais, de monitoramento, para a customização da análise de dados agregados, assim como para a formatação de dados que permita a exportação para os sistemas de informação em saúde oficiais.

\section{Conclusões}

As questões relativas à interoperabilidade e à coerência semântica são mais relevantes para os sistemas de informação em saúde do que para qualquer outro setor econômico da sociedade. Isto ocorre em função da necessidade de manutenção de registro de saúde do indivíduo de forma longitudinal durante toda a sua vida. No entanto, a extrema dinâmica em termos conceituais, espaciais e temporais das ações em saúde requer um alto nível de diversidade entre os sistemas de informação em saúde, adequados a diferentes serviços e propósitos. Sistemas de informação tradicionais, baseados em modelos de dados de um nível, que se encaixam às necessidades de praticamente qualquer outra atividade econômica da sociedade humana, estão sendo usados há 50 anos no setor saúde e ainda não foram capazes de gerar um registro de saúde centrado no cidadão, interoperável e semanticamente coerente. Ao longo dos últimos 20 anos, especificações de modelagem multinível têm sido desenvolvidas, a fim de superar estes desafions. Durante esse processo de desenvolvimento, tornouse evidente que a verdadeira interoperabilidade só será alcançada se as especificações de modelagem multinível estejam disponíveis abertamente. A implementação das especificações de modelagem multinível em software livre tem o potencial de aumentar a concorrência para o desenvolvimento de software de boa qualidade, o que é fundamental para o setor de saúde. Este artigo apresentou o estado da arte das especificações de modelagem multinível em software livre que estão disponíveis atualmente, o que demonstra a possibilidade prática de desenvolvimento de sistemas de informação em saúde de código aberto, com base em modelagem multinível. Assim, ao contribuir para estes projectos, a comunidade de software livre pode ajudar a melhorar a qualidade dos sistemas de saúde de uma forma global. 


\section{Referências}

1. Hudson DL, Cohen ME. Uncertainty and complexity in personal health records. Conf Proc IEEE Eng Med Biol Soc 2010; 2010: 6773-6.

2. Kelley J. The interoperability hang-up. When it comes to information exchange, how should precede what. Health Manag Technol 2011;32(2): 32, 34.

3. Haughton J. Look up: the right EHR may be in the cloud. Major advantages include interoperability and flexibility. Health Manag Technol 2011; 32(2): 52.

4. Hammond WE, Bailey C, Boucher P, Spohr M, Whitaker P. Connecting information to improve health. Health Aff (Millwood) 2010; 29(2): 284-8.

5. Garde S, Chen R, Leslie H, Beale T, McNicoll I, Heard S. Archetype-based knowledge management for semantic interoperability of electronic health records. Stud Health Technol Inform 2009; 150: 1007-11.

6. Haux R. Medical informatics: past, present, future. Int J Med Inform 2010; 79(9): $599-610$

7. Blobel B. Comparing concepts for electronic health record architectures. Stud Health Technol Inform 2002; 90: 209-14.

8. Cantiello J, Cortelyou-Ward KH. The American Recovery and Reinvestment Act: lessons learned from physicians who have gone electronic. Health Care Manag (Frederick) 2010; 29(4): 332-8

9. Michelsen L, Pedersen SS, Tilma HB, Andersen SK. Comparing different approaches to two-level modelling of electronic health records. Stud Health Technol Inform 2005; 116: 113-8.

10. Eccles M, Mason J. How to develop cost-conscious guidelines. Health Technol Assess 2001; 5(16): 1-69.

11. Kalra D, Beale T, Heard S. The openEHR Foundation. Stud Health Technol Inform 2005; 115: 153-73.

12. Kohl CD, Garde S, Knaup P. Facilitating secondary use of medical data by using openEHR archetypes. Stud Health Technol Inform 2010; 160(Pt 2): 1117-21.

13. Garde S, Knaup P, Hovenga E, Heard S. Towards semantic interoperability for electronic health records. Methods Inf Med 2007; 46(3): 332-43.

14. Ammenwerth E, Shaw NT. Bad informatics can kill: is evaluation the answer? Methods Inf Med 2005; 44(1): 1-3.

15. Chen R, Klein G. The openEHR Java reference implementation project. Stud Health Technol Inform 2007; 129(Pt 1): 58-62.

16. Kashfi H. An openEHR-based clinical decision support system: a case study. Stud Health Technol Inform 2009; 150: 348.

17. Martinez-Costa C, Menarguez-Tortosa M, Fernandez-Breis JT. An approach for the semantic interoperability of ISO EN 13606 and OpenEHR archetypes. J Biomed Inform 2010; 43(5): 736-46.

18. Almeida F, Oliveira J, Cruz J. Open standards and open source: enabling interoperability. Int J Soft Eng App 2011; 2(1):10.5121/ijsea.2011.2101.
19. Dutton WH. Key enablers for eTransformation? eID, interoperability and open source. Eur J ePractice 2009; 6:2

20. Reynolds CJ, Wyatt JC. Open Source, open standards, and health care information systems. J Med Internet Res 2011;13(1):e24.

21. Barretto SA, Warren J, Goodchild A, Bird L, Heard S, Stumptner M. Linking guidelines to Electronic Health Record design for improved chronic disease management. AMIA Annu Symp Proc 2003; 66-70.

22. Beale T, Heard S. openEHR Architecture overview. London 2008; openEHR Foundation. 23. Neeser AE. XML: The open source solution to interoperability. Open Lib Class 2009; 1(2). Available at http://www.infosherpas.com/ojs/index.php/openandlibraries/ article/view/31/40. Accessed on Mar 6th, 2011.

24. Paterson T, Law A. An XML transfer schema for exchange of genomic and genetic mapping data: implementation as a web service in a Taverna workflow. BMC Bioinformatics 2009; 10:252.

25. Seibel PN, Krüger J, Hartmeier S, Schwarzer K, Löwenthal K, Mersch H, Dandekar T, Giegerich R. XML Schemas for common bioinformatic data types and their application in workflow systems. BMC Bioinformatics 2006; 7:490.

26. Norlin C, Kerr LM, Rocha RA. Using clinical questions to structure the content of a webbased information resource for primary care physicians. AMIA Symp Proc 2009; 482-6.

27. Zhao L, Lee KP, Hu J. Generating XML Schemas for DICOM structured reporting templates. J Am Med Inform Assoc 2005;12:72-83.

28. Gao S, Mioc D, Yi X, Anton F, Oldfield E, Coleman DJ. Towards web-based representation and processing of health information. Int J Health Geographics 2009; 8:3.

29. Hägglund M, Scandurra I, Moström D, Koch S. Bridging the gap: a virtual health record for integrated home care. Int J Integr Care 2007; 7:26.

30. Qian Y, Tchuvatkina O, Spidlen J, Wilkinson P, Gasparetto M, Jones AR, Manion FJ, Scheuermann RH, Sekaly RP, Brinkman RR. FuGEFlow: data model and markup language for flow cytometry. BMC Bioinformatics 2009; 10:184.

31. Hulse NC, Rocha RA, Del Fiol G, Bradshaw RD, Hanna TP, Roemer LK. KAT: A flexible XML-based knowledge authoring environment. J Am Med Inform Assoc 2005;12:418-430.

32. Rinner C, Janzek-Hawlat S, Sibinovic S, Duftschmid G. Semantic validation of standard-based electronic health record documents with W3C XML schema. Methods Inf Med 2010;49(3):271-80

33. Maldonado JA, Moner D, Bosca D, Fernandez-Breis JT, Angulo C, Robles M. LinkEHREd: a multi-reference model archetype editor based on formal semantics. Int J Med Inform 2008; 78(8): 559-70.

34. Sundvall E, Qamar R, Nystrom M, Forss M, Petersson $H$, Karlsson D, Ahlfeldt $H$, Rector A. Integration of tools for binding archetypes to SNOMED CT. BMC Med Inform Decis Mak 2008; 8 Suppl 1: S7.

35. D'Antoni AV, Zipp GP, Olson VG, Cahill TF. Does the mind map learning strategy facilitate information retrieval and critical thinking in medical students? BMC Med Educ 2010; 10: 61 . 